\title{
FACTORES CONDICIONANTES DE LA LACTANCIA EN RECIÉN NACIDOS SANOS DEL HOSPITAL UNIVERSITARIO CLÍNICA SAN RAFAEL (HUCSR)
}

\author{
Rida M. Bejarano R., MD. ${ }^{1}$, Mónica Fuentes, MD. ${ }^{2}$, \\ Octavio Castellanos, MD. ${ }^{3}$, José Manuel Nieto, MD. ${ }^{3}$ Laura Vargas, $M D .{ }^{4}$
}

\begin{abstract}
${ }^{1}$ Pediatra, Hospital Universitario Clínica San Rafael, Bogotá D.C., Colombia. ${ }^{2}$ Epidemióloga, Residente III de Pediatría, Universidad Militar Nueva Granada, Programa Hospital Universitario Clínica San Rafael, Bogotá. D.C., Colombia. ${ }^{3}$ Residentes de III año de Pediatría, Universidad Militar Nueva Granada, Programa Hospital Universitario Clínica San Rafael, Bogotá. D.C., Colombia.

${ }^{4}$ Residente de II año de Pediatría, Universidad Militar Nueva Granada, Programa Hospital universitario Clínica San Rafael, Bogotá. D.C., Colombia.
\end{abstract}

\begin{abstract}
Resumen
Tradicionalmente se han descrito factores asociados a la Lactancia que varían en las diferentes culturas. En Colombia, los indicadores de prevalencia de la Lactancia materna exclusiva y duración de la Lactancia están lejos de las recomendaciones establecidas por la Organización Mundial de la Salud (OMS). El Departamento de Pediatría del Hospital Universitario Clínica San Rafael, teniendo en cuenta los beneficios que la leche materna tiene, ha venido trabajando en los últimos años en pro de rescatar la lactancia como una práctica cultural adecuada. En respuesta a esta necesidad creó en Agosto de 2004 el programa institucional de Lactancia "Primero los niños, niñas y adolescentes", a partir del cual surgieron varios interrogantes acerca del comportamiento de la lactancia en nuestro medio, que dieron origen al presente trabajo de investigación. Se diseñó un estudio de cohorte prospectivo realizado mediante la aplicación de una encuesta a 372 madres de recién nacidos sanos en postparto. La cohorte se reclutó entre julio de 2007 y junio de 2008 y se siguió hasta los 24 meses de edad del bebé con el fin de determinar la duración de la lactancia, sus factores condicionantes y las causas más frecuentes de su abandono. Se estableció la salida de los pacientes del estudio al cumplir 24 meses (recomendación de la OMS de la duración de lactancia) o al documentar su abandono antes de los 24 meses. Se encontró una lactancia materna exclusiva a los 6 meses de 14.7\% y una duración acumulada de 14 meses para la población analizada.
\end{abstract}

Palabras clave: Lactancia materna, factores condicionantes.

\section{FACTORS CONDITIONING THE HEALTHY NEWBORNS BREASTEEDING IN HOSPITAL UNIVERSITARIO CLÍNICA SAN RAFAEL (HUCSR)}

\begin{abstract}
Summary
Traditionally, factors associated with breastfeeding which vary according to the different cultures have been described. In Colombia, the indicators of exclusive breastfeeding prevalence and breastfeeding duration are very far from the recommendations settled by the World Health Organization (WHO). The Pediatrics Department of the Hospital Universitario Clínica San Rafael, taking into account the
\end{abstract}

Correspondencia: ridabejarano@gmail.com

Recibido: julio 7 de 2011 Aceptado: diciembre 5 de 2011 
benefits provided by the breast milk, have been working for the last years in favor of rescuing the breastfeeding as a suitable cultural practice. In response to this need, in August 2004, it created the Breastfeeding institutional program "First boys, girls and adolescents", form which various questions emerged regarding the breastfeeding behavior in our environment, which gave rise to the present research work. A cohort, prospective study was designed, conducted by administering a survey to 372 mothers of healthy newborns in post partum. The cohort was recruited between July 2007 and June 2008 and was followed-up until the infants were 24 months of age with the aim of determining the breastfeeding duration, its conditioning factors and the most frequent causes for its drop-out. The infants were to be withdrawed from the study when they completed 24 months of age (Breastfeeding duration recommended by the $\mathrm{WHO}$ ) or upon documenting their drop-out before 24 months of age. $14.7 \%$ of exclusive breastfeeding at 6 months and a cumulative duration of 14 months were found for the examined population.

Key words: Breastfeeding, conditioning factors.

\title{
FATORES CONDICIONANTES DA AMAMENTAÇÃO EM RECÉM-NASCIDOS SAUDÁVEIS DO HOSPITAL UNIVERSITÁRIO CLÍNICA SAN RAFAEL (HUCSR)
}

\author{
Resumo
}

\begin{abstract}
Tradicionalmente foram descrito fatores associados à amamentação que variam nas diferentes culturas. Na Colômbia, os indicadores de prevalência da amamentação materna exclusiva e duração da amamentação estão longe das recomendações estabelecidas pela Organização Mundial da Saúde (OMS). O Departamento de Pediatria do Hospital Universitário Clínica San Rafael, tendo em conta os benefícios que o leite materno tem, vem trabalhando nos últimos anos em prol de resgatar a amamentação como uma prática cultural adequada. Em resposta a esta necessidade criou-se em agosto de 2004 o programa institucional de Amamentação "Primeiro as crianças e adolescentes", a partir do qual surgiram várias dúvidas sobre o comportamento da amamentação em nosso meio que deram origem a este trabalho de pesquisa. Foi desenhado um estudo de cooorte prospectivo realizado mediante a aplicação de um questionário a 372 mães de recém nascido sãos em pós-parto. A população foi recrutada entre julho de 2007 e junho de 2008 e foi seguida até os 24 meses de idade do bebê com o objetivo de determinar a duração da amamentação, seus fatores condicionantes e as causas mais frequentes de seu abandono. Foi estabelecida a saída dos pacientes do estudo ao completar 24 meses (recomendação da OMS da duração da lactação) ou ao documentar seu abandono antes dos 24 meses. Encontrou-se uma lactação materna exclusiva aos 6 meses de 14.7\% e uma duração acumulada de 14 meses para a população analisada.
\end{abstract}

Palavras chave: Amamentação materna, fatores condicionantes.

\section{Introducción}

El HUCSR es una Institución de alto nivel de complejidad que atiende pacientes provenientes principalmente del suroriente de Bogotá. El Departamento de Pediatría del HUCSR, que ha venido trabajando de la mano con el Programa Madre Canguro en pro de rescatar la lactancia como práctica cultural adecuada, creó en Agosto de 2004 el programa institucional de Lactancia "Primero los niños, niñas y adolescentes", a partir del cual surgieron varios interrogantes acerca del comportamiento de la lactancia en nuestro medio, que dieron origen al presente trabajo de investigación.

Los objetivos fueron:

a) Describir las características socio-demográficas de la población encuestada.

b) Determinar la duración de la lactancia en recién nacidos sanos del HUCSR.

c) Determinar la relación existente entre factores condicionantes de la lactancia (edad de la madre, 
estrato socioeconómico, escolaridad, estado civil, actividad laboral, experiencia anterior de la madre con la lactancia y el inicio temprano de lactancia) y su duración a los 6, 12 y 24 meses de edad del lactante, en madres de bebés sanos nacidos en el HUCSR.

d) Establecer las causas más frecuentes de su abandono.

\section{Materiales y métodos}

Se realizó un estudio de cohorte prospectivo mediante la aplicación, previo consentimiento informado, de una encuesta (ver anexo) a las madres de recién nacidos sanos en alojamiento conjunto. La cohorte se reclutó entre julio de 2007 y junio de 2008 y se siguió de manera telefónica a los 6, 12, 18 y 24 meses de edad del bebé. Se estableció la salida de los pacientes del estudio al cumplir 24 meses (recomendación de la OMS) o al documentar su abandono antes de los 24 meses. Se consideró abandono la no administración de leche materna en ninguna cantidad. El estudio se realizó con la participación de residentes de Pediatría de diferentes niveles de la Universidad Militar Nueva Granada, Programa HUCSR.

El tamaño de la muestra se calculó en el programa Episet, con un nivel de confianza del 95\% teniendo en cuenta la prevalencia en Colombia de la lactancia hasta los 24 meses de edad (31.2\% según la ENDS de 2005. 10). La muestra a tomar fue definida en 329 pacientes, sin embargo se presupuestó un índice de pérdidas del 10\% llegándose a una muestra final de 362 pacientes.

Se incluyeron madres con recién nacidos sanos cuyo parto fue atendido en el HUCSR, que permanecieron en alojamiento conjunto y fueron dados de alta con la madre; se excluyeron del estudio las madres de recién nacidos trasladados a la unidad neonatal, de recién nacidos con contraindicaciones absolutas para la lactancia, madres con antecedentes de mortinatos, óbitos fetales o con limitaciones físicas para contestar la encuesta.

Para la construcción de la base de datos se utilizó el programa Excel versión 2003 y el programa STATA IC 10.1 licenciado para la depuración, codificación y procesamiento de las variables.

Hasta el momento de la segunda encuesta se presentaron 188 retiros (50\%) de la cohorte debido a cambio de teléfono o domicilio, retiros voluntarios, números telefónicos dañados o imposibilidad de contacto. Para disminuir este porcentaje se comenzó a registrar al menos 3 números de teléfono y se volvió a llamar a todas las participantes excepto a quienes habían manifestado su retiro voluntariamente, logrando recuperar un $5 \%$ de las pérdidas.

\section{Resultados}

Se encuestaron 372 mujeres cuya media de edad fue de 25 años, $75.3 \%$ pertenecían a los estratos 1 y 2 , (77.4\%) contaban con una relación estable, (29.8\%) habían alcanzado algún grado de educación superior, (46.8\%) tenían algún tipo de actividad laboral, (49.5\%) tenían al menos un hijo y de ellas el (81.5\%) consideraban tener una buena experiencia previa en relación a la lactancia. De las 184 mujeres con antecedente de embarazos previos, (29\%) contestaron haber tenido lactancia exclusiva durante un periodo mayor de 6 meses.

Se logró establecer que solo para el caso del estado civil existe una asociación estadísticamente significativa entre el grupo de mujeres que tenían una unión estable y la persistencia de la lactancia materna a los 6 y 12 meses de edad del lactante.

En la tercera encuesta telefónica de los 11 a los 16 meses, no se encontró relación significativa entre las variables a estudio y la duración de la lactancia materna.

Al concluir el seguimiento de la cohorte a los 24 meses se encontró una relación significativa entre colocar al recién nacido tempranamente al pecho y la duración de la lactancia, concordante con la política establecida por el HUCSR de iniciar de lactancia poco tiempo después del nacimiento.

La duración de la lactancia para la primera consulta telefónica fue de 5,1 meses, así como de 2,7 y 2,6 meses para la segunda y tercera consultas telefónicas respectivamente. Se evidenció un aumento en la duración de la lactancia para la cuarta consulta telefónica que se explica por el esfuerzo para recuperar la cohorte, con una duración acumulada de lactancia de 14 meses para la población analizada. (Tabla No. 1 Duración de la Lactancia en meses). 
Tabla 1. Duración de la Lactancia en meses

\begin{tabular}{|l|c|}
\hline \multicolumn{1}{|c|}{ PERIODO } & Duración en promedio \\
\hline Primera consulta & 5.1 meses \\
\hline Segunda consulta telefónica & 2.7 meses \\
\hline Tercera consulta telefónica & 2.6 meses \\
\hline Cuarta consulta telefónica & 3.6 meses \\
\hline TOTAL (Duración acumulada) & 14 meses \\
\hline
\end{tabular}

Los resultados principales en la distribución de la lactancia en los 6 primeros meses según las definiciones de la OMS fueron (Tabla No.2. Distribución de los tipos de lactancia en los primeros 6 meses):

Lactancia materna exclusiva: pecho como único alimento, pudiendo recibir además vitaminas, minerales o medicamentos. $14.7 \%$

Lactancia materna predominante: pecho más agua, zumos, té, SRO (solución de rehidratación oral). $20.9 \%$

Lactancia materna parcial: pecho más otra leche o papilla $16.9 \%$.

Tabla 2. Distribución de los principales tipos de lactancia en los primeros 6 meses

\begin{tabular}{|l|l|l|}
\hline \multicolumn{1}{|c|}{ Tipo } & \multicolumn{1}{c|}{ N } & \multicolumn{1}{c|}{ Porcentaje } \\
\hline Lactancia exclusiva & 55 & $14,78 \%$ \\
\hline Lactancia parcal & 63 & $16,94 \%$ \\
\hline Lactancia predominante & 78 & $20,97 \$$ \\
\hline & & \\
\hline \multicolumn{1}{|c|}{ CAUSAS } & Frecuencia & Porcentaje \\
\hline Por indicación médica & 110 & $21.19 \%$ \\
\hline Otras razones & 108 & $20.81 \%$ \\
\hline Por insuficiente cantidad de leche & 95 & $18.30 \%$ \\
\hline
\end{tabular}

La causa más frecuente de abandono de la lactancia encontrada en el estudio fue la variable "por indicación médica". Las indicaciones médicas establecidas en la literatura para la suspensión del amamantamiento se pueden resumir en infecciones de la madre, administración de ciertos medicamentos a la madre y algunas condiciones clínicas del bebé. En la encuesta se indagó específicamente por estas 3 situaciones, las cuales no estuvieron presentes en la población analizada.

Ahora bien, a pesar de haberse definido en la encuesta 15 posibles motivos de abandono de la lactancia, se encontró en segundo lugar: "otras causas". Los siguientes motivos de abandono encontrados después de "indicación médica" y "otras causas" fueron en su orden: insuficiente cantidad de leche, desconocimiento, no le gusta al bebé, ingreso al trabajo y porque es doloroso.

\section{Conclusiones}

Los resultados de este estudio en cuanto a la prevalencia de la lactancia materna exclusiva y duración de la Lactancia son similares a los datos encontrados en otros estudios a nivel nacional. En lo que se refiere a los motivos de abandono llama la atención el motivo "indicación médica”, el cual, para la población analizada, no tiene relación con las causas descritas en la literatura para la suspensión de la lactancia. Surge la inquietud de si "la indicación médica" de suspender la lactancia estuvo debidamente justificada. Valdría la pena indagar más sobre este aspecto en estudios posteriores.

Vemos con extrañeza el segundo motivo de abandono definido como "otras causas" que pudiera corresponder a motivos diferentes a los tradicionalmente conocidos y que también puede dar origen a otra pregunta de investigación.

Finalmente pensamos que es necesario establecer estrategias nacionales que permitan crear conciencia en el equipo de salud para que se insista en la lactancia materna como la mejor opción para la alimentación de nuestros pacientes. Podríamos comenzar con los estudiantes de pre y postgrado de las diferentes disciplinas del área de la salud.

Tabla 3. Distribución causas de abandono de la Lactancia

\begin{tabular}{|l|c|c|}
\hline Desconocimiento & 77 & $14.84 \%$ \\
\hline Porque no le gusta al bebé & 39 & $7.51 \%$ \\
\hline Ingresó al trabajo & 30 & $5.78 \%$ \\
\hline Porque es doloroso & 18 & $3.47 \%$ \\
\hline
\end{tabular}

\section{Agradecimientos}

Agradecemos muy especialmente a la Dra. Olga Lucía Pinto, pediatra, epidemióloga y al Dr. Fernando Suárez, Genetista, epidemiólogo quienes nos asesoraron desde el punto de vista metodológico para la realización del estudio. También a la Dra. Clemencia Mayorga por su permanente motivación para la realización de trabajos de investigación que aporten conocimientos para el mejoramiento de las condiciones de salud de los niños, niñas y adolescentes colombianos. 


\section{Anexo 1}

FECHA DE ENCUESTA:

ENCUESTA No 2007

Para las siguientes preguntas seleccione en el recuadro de acuerdo a su respuesta.

\section{DATOS GENERALES}

\section{IDENTIFICACIÓN DE LA MADRE}

1. Nombre de la madre:

3. Documento de Identificación:

2. Edad:

4. Tipo Doc:

4.1. $\mathrm{RC}$

4.2. TI

4.3. CC

4.4. Otro

5. Dirección domicilio:

6. EPS:

7. Teléfonos $\square$ Teléfono 1 :

$\square$ Teléfono 2:

$\square$ Teléfono 3:

\section{DATOS SOCIOECONÓMICOS}

\section{GRADO DE ESCOLARIDAD MATERNA}

$\square$ 1. Analfabeta

$\square$ 2. Primaria incompleta

$\square$ 3. Primaria completa

4. Secundaria incompleta
5. Secundaria completa

$\square$ 6. Superior incompleta

$\square$ 7. Superior completa

8. Postgrados

III. ESTADO CIVIL DE LA MADRE
$\square$ 1. Soltera
$\square$ 4. Separada
2. Casada
$\square$ 5. Divorciada
3. Unión libre
$\square$ 6. Viuda

IV. ESTRATO SOCIOECONÓMICO (según recibo de servicios públicos)
1. Estrato uno
4. Estrato cuatro
2. Estrato dos
5. Estrato cinco
3. Estrato tres
6. Estrato seis

V. MADRE TRABAJA

$\square$ 1. Si $\square$ 1.1. Tiempo completo $\square$ 2. No

$\square$ 1.2. Medio tiempo

$\square$ 1.3. Otro

\section{DATOS DE EMBARAZOS ANTERIORES}

VI. NÚMERO DE HIJOS ANTERIORES (sin contar el actual)

1. Ninguno

2. Uno
3. Dos

4. Tres
5. Cuatro

6. Cinco o más

Edad de último hijo vivo (sin contar el actual)

Si ha tenido hijos anteriores, responder las VII a X. Si la respuesta es ninguno, pasar a pregunta XI. 
VII. ¿ASISTIÓ A CURSO PSICOPROFILÁCTICO EN EMBARAZOS ANTERIORES?
1. $\mathrm{Si}$
2. No

VIII. EXPERIENCIA CON LA LACTANCIA ANTERIOR
1. Muy buena
$\square$ 4. Mala
$\square$ 2. Buena
$\square$ 5. Muy mala
$\square$ 3. Regular
$\square$ 6. Pésima

IX. LACTANCIA EXCLUSIVA CON EL HIJO ANTERIOR (solo leche materna, sin ningún otro alimento)
$\square$ 1. Menos de un mes
$\square$ 5. Cuatro a cinco meses
2. Uno a dos meses
$\square$ 6. Cinco a seis meses
3. Dos a tres meses
$\square$ 7. Más de seis meses
4. Tres a cuatro meses
Cuantos meses?

\section{LACTANCIA MÁS ALIMENTACIÓN COMPLEMENTARIA CON EL HIJO ANTERIOR}
1. Menos de dos meses
$\square$ 2. Dos a cuatro meses
3. Cuatro a seis meses
$\square$ 4. Seis a ocho meses
$\square$ 5. Ocho a diez meses
$\square$ 6. Diez a doce meses
$\square$ 7. Doce a catorce meses
$\square$ 8. Catorce a dieciséis meses
$\square$ 9. Dieciséis a dieciocho meses
$\square$ 10. Dieciocho a veinte meses
$\square$ 11. Veinte a veintidós meses
$\square$ 12. Veintidós a veinticuatro meses
$\square$ 13. Mas de veinticuatro meses
¿Cuántos meses?

\section{DATOS DE EMBARAZO Y PARTO ACTUAL}

\section{XI. ¿ASISTIÓ A CURSO PSICOPROFILÁCTICO EN EMBARAZO QUE ACABA DE TERMINAR?}
1. $\mathrm{Si}$
2. No

XII. TIPO DE PARTO
1. Vaginal
2. Instrumentado
3. Cesárea

XIII.- ¿SE LE COLOCÓ A SU HIJO EN EL PECHO INMEDIATAMENTE DESPUÉS DE NACER?
1. $\mathrm{Si}$
2. No

XIV.- ¿AL CUÁNTO TIEMPO DE HABER NACIDO SU BEBÉ SE INICIÓ LA LACTANCIA?
1. Antes de $1 / 2$ Hora
2. Entre $1 / 2$ y 1 hora
3. Más de 1 hora

Si su respuesta es 3 , ¿por qué?

XV. ¿ ¿LO ÚLTIMO QUE TOMÓ SU BEBÉ FUE?
1. Leche materna
$\square$ 2. Otra leche
3. Otro líquido
4. Otro alimento
$\square$ 5. Mixto (leche materna y leche f'Ormula)
$\square$ 6. No sabe

$\square$ 1. Por demora en pasarle el bebé

$\square$ 2. Por algún procedimiento a la madre

$\square$ 3. Por efecto de la anestesia materna

$\square$ 4. Porque no le informaron sobre iniciar la lactancia 
PRIMERA CONSULTA TELEFÓNICA

CONSULTA 6 MESES

FECHA:

XVI. ¿EL DÍA DE AYER SU HIJO TOMÓ?
1. Leche materna
2. Otra leche
3. Otro líquido
$\square$ 4. Otro alimento

XVII.- ¿POR QUE DEJÓ DE LACTAR?

Especifique

$\square$ 1. Enfermedad de la madre
$\square$ 2. Enfermedad de recién nacido
$\square$ 3. Porque madre recibe medicamentos
$\square$ 4. Porque madre planifica
$\square$ 5. Por indicación médica
$\square$ 6. Situación estresante para la madre
$\square$ 7. Ingreso al trabajo
$\square$ 8. Desconocimiento
$\square$ 9. Porque no le gusta a la mama
$\square$ 10. Porque no le gusta al bebé
$\square$ 11. Porque el niño queda con hambre
$\square$ 12. Porque carece de valor nutricional
$\square$ 13. Por insuficiente cantidad de leche
$\square$ 14. Porque es doloroso
$\square$ 15. Porque se le crecen los senos
$\square$ 16. Otra razón

\section{RETIRO DEL ESTUDIO}

$\square$ 1. No contesta
$\square$ 2. Teléfono dañado
$\square$ 3. Cambio de domicilio
$\square$ 4. Cambio de teléfono
$\square$ 5. Retiro voluntario

$\square$ 6. Se niega

$\square$ 7. Muerte de la madre

$\square$ 8. Muerte del hijo

$\square$ 9. Otro

$\square$ 5. Retiro voluntario

¿Cuál?

\section{SEGUNDA CONSULTA TELEFÓNICA}

CONSULTA 7 A 10 MESES

FECHA:

\section{XIX. ¿EL DÍA DE AYER SU HIJO TOMÓ?}

1. Leche materna

3. Otro líquido

XX. ¿POR QUÉ DEJÓ DE LACTAR? $\square$ 2. Otra leche

$\square$ 4. Otro alimento

1. Enfermedad de la madre

$\square$ 2. Enfermedad de recién nacido

$\square$ 3. Porque madre recibe medicamentos

$\square$ 4. Porque madre planifica

$\square$ 5. Por indicación médica

$\square$ 6. Situación estresante para la madre

$\square$ 7. Ingreso al trabajo

$\square$ 8. Desconocimiento 
9. Porque no le gusta a la mamá

10. Porque no le gusta al bebé

11. Porque el niño queda con hambre

12. Porque carece de valor nutricional

13. Por insuficiente cantidad de leche

14. Porque es doloroso

15. Porque se le crecen los senos

16. Otra razón

\section{RETIRO DEL ESTUDIO}

$\square$ 1. No contesta
$\square$ 2. Teléfono dañado
$\square$ 3. Cambio de domicilio
$\square$ 4. Cambio de teléfono
$\square$ 5.

$\square$ 9. Otro

5. Retiro voluntario

Cuál?

\section{TERCERA CONSULTA TELEFÓNICA}

CONSULTA 12 A 16 MESES

FECHA:

\section{XXII.EL DÍA DE AYER SU HIJO TOMÓ}
1. Leche materna
2. Otra leche

XXIII. ¿POR QUÉ DEJO DE LACTAR? $\square$ 3. Otro líquido

$\square 4$ Otro alimento

Especifique

1. Enfermedad de la madre

$\square$ 2. Enfermedad de recién nacido

$\square$ 3. Porque madre recibe medicamentos

$\square$ 4. Porque madre planifica

$\square$ 5. Por indicación médica

$\square$ 6. Situación estresante para la madre

$\square$ 7. Ingreso al trabajo

$\square$ 8. Desconocimiento

9. Porque no le gusta a la mamá

10. Porque no le gusta al bebé

$\square$ 11. Porque el niño queda con hambre

$\square$ 12. Porque carece de valor nutricional

$\square$ 13. Por insuficiente cantidad de leche

$\square$ 14. Porque es doloroso

$\square$ 15. Porque se le crecen los senos

16. Otra razón

\section{RETIRO DEL ESTUDIO}

\begin{tabular}{l}
$\square$ 1. No contesta \\
$\square$ 2. \\
$\square$ 3eléfono dañado \\
$\square$ 4. \\
$\square$ 4. Cambio de domicilio \\
$\square$ 5. \\
\hline Retiro voluntario
\end{tabular}

6. Se niega

$\square$ 7. Muerte de la madre

$\square$ 8. Muerte del hijo

$\square$ 9. Otro ¿Cuál? 
CUARTA CONSULTA TELEFÓNICA

FECHA:

\section{XXV.EL DÍA DE AYER SU HIJO TOMÓ}
1. Leche materna
3. Otro líquido
2. Otra leche
$\square$ 4. Otro alimento

XXVI. ¿POR QUÉ DEJÓ DE LACTAR?

Especifique

1. Enfermedad de la madre

$\square$ 2. Enfermedad de recién nacido

$\square$ 3. Porque madre recibe medicamentos

$\square$ 4. Porque madre planifica

$\square$ 5. Por indicación médica

$\square$ 6. Situación estresante para la madre

$\square$ 7. Ingreso al trabajo

$\square$ 8. Desconocimiento

$\square$ 9. Porque no le gusta a la mama

$\square$ 10. Porque no le gusta al bebe

$\square$ 11. Porque el niño queda con hambre

$\square$ 12. Porque carece de valor nutricional

$\square$ 13. Por insuficiente cantidad de leche

$\square$ 14. Porque es doloroso

$\square$ 15. Porque se le crecen los senos

$\square$ 16. Otra razón

\section{RETIRO DEL ESTUDIO}

1. No contesta

2. Teléfono dañado

3. Cambio de domicilio

4. Cambio de teléfono

5. Retiro voluntario
6. Se niega

$\square$ 7. Muerte de la madre

$\square$ 8. Muerte del hijo

$\square$ 9. Otro

¿Cuál? 


\section{Referencias}

1. Comité de Lactancia Materna de la AEP. Monografías de la AEP. No.5. Lactancia Materna. Guía para profesionales. Barcelona. Febrero de 2004.

2. Kramer Ms, Kakuma R. Optimal duration of exclusive breastfeeding. The Cochrane database of Systematic Reviews 2002.

3. American Academy of Pediatrics Policy. Breastfeeding and the use of Human Milk. Pediatrics. Feb 2005; 115(2):496-506.

4. Bejarano R, Acosta G, Mayorga C, Castro C. Lactancia en el Hospital: "Primero los niños". 2005.

5. Encuesta Nacional sobre Demografía y Salud, Bogotá, 1990, 1995 y 2000, Informe oficial de Colombia sobre el alcance de las metas declaradas para el 2000 en la Cumbre de Jefes de Países en 1990, Bogotá, 2001.

6. ICBF. Encuesta Nacional sobre Demografía y Salud, Bogotá, $2005 ; 247-286$
7. Álvarez Uribe M, González Zapata L. Feeding practices among rural families of Medellín, Colombia. Archivos Latinoamericanos de Nutrición. Mar 2002: 52 (1):55-62.

8. UNICEF. Instituciones Amigas de la Mujer y la Infancia en el Marco de los Derechos. Manual para su aplicación. "Por el interés superior del Niño". Abr 2005; 59-60.

9. Ministerio de la protección Social, Viceministerio de Salud y Bienestar. Instituto Colombiano de Bienestar Familiar, Plan decenal para la Promoción, Protección y Apoyo a la Lactancia Materna 1998-2008. Lineamientos para la programación de actividades a realizar durante la $13^{\mathrm{a}}$ semana mundial de la Lactancia.

10. Encuesta sobre Salud Sexual y Reproductiva. Profamilia y Ministerio de la Protección Social. Diciembre de 2005. 